\title{
Unified Picture of Anionic Redox in Li/Na-ion Batteries
}

\author{
Mouna Ben Yahia ${ }^{1,2}$, Jean Vergnet ${ }^{2,3}$, Matthieu Saubanère ${ }^{1,2, *}$, and Marie-Liesse Doublet ${ }^{1,2 *}$ \\ ${ }^{1}$ Institut Charles Gerhardt, CNRS - Université de Montpellier, \\ Place Eugène Bataillon, 34095 Montpellier, France \\ ${ }^{2}$ Réseau Français sur le Stockage Électrochimique de l'Énergie (RS 2 E), FR5439, Amiens, France and \\ ${ }^{3}$ Collège de France, Chimie du Solide et de l'Énergie, UMR CNRS 8260, Paris, France.
}

\begin{abstract}
Anionic redox in Li-rich and Na-rich Transition Metal Oxides (Arich-TMOs) has emerged as a new paradigm to increase the energy density of rechargeable batteries. Ever since, numerous electrodes delivering anionic extra-capacity beyond the theoretical cationic capacity were reported. Unfortunately, most often the anionic capacity achieved in charge is partly irreversible in discharge. A unified picture of anionic redox in Arich-TMOs is here designed to identify the electronic origin of this irreversibility and to propose new directions to improve the cycling performance of the electrodes. The electron localization function (ELF) is introduced as a holistic tool to unambiguously locate the oxygen lone-pairs in the structure and follow their participation in the redox activity of Arich-TMOs. The charge-transfer gap of transition metal oxides is proposed as the pertinent observable to quantify the amount of extra-capacity achievable in charge and its reversibility in discharge, irrespectively of the material chemical composition. From this generalized approach, we conclude that the reversibility of the anionic capacity is limited to a critical number of O-hole per oxygen, $h^{O} \leq 1 / 3$.
\end{abstract}

\section{GENERAL CONTEXT}

Increasing the energy density of cathode materials is a central goal of ongoing research in $\mathrm{Li} / \mathrm{Na}$-ion batteries. This implies improving simultaneously the material potential and capacity. Whereas potential can be easily tuned through an appropriate choice of the redox centre of the electrochemical reaction, $[1,2]$ capacity is more challenging to increase without penalizing the material structure. Thanks to the cumulative cationic and anionic activity recorded in $\mathrm{Li} / \mathrm{Na}$-rich transition metal oxides (Arich-TMOs), [3-9] anionic redox emerged as a new paradigm to increase energy density. However, extra-capacities due to anions generally lead to structural instabilities resulting in significant irreversibility in the first cycle, [10] capacity and potential fading upon cycling, [11] cationic migrations [12, 13] and oxygen loss. [6, 12, 14-16] This yields continuous decomposition of the electrode upon cycling which constitutes a major drawback for practical applications. The opportunity to play with $\mathrm{M} / \mathrm{M}$ ' chemical substitutions [7], structural dimensionality [9] or cation-disorder [17] has raised hopes that a winning combination can suppress these irreversibilities, hence enabling long-term cycling for high-energy-density electrodes. Recent examples supporting this line were reported by different groups and summarized in several reviews. [18-20] Among them, the tri-dimensional $\beta$ - $\mathrm{Li}_{2} \mathrm{IrO}_{3}$ model electrode [9] combines improved cycling performance and a record capacity of $2.5 \mathrm{Li} / \mathrm{Ir}$; the lighter $\mathrm{Li}_{1.3} \mathrm{Nb}_{0.3} \mathrm{Mn}_{0.4} \mathrm{O}_{2}$ electrode [7] displays the highest first-charge capacity $(>300$ $\mathrm{mAh} / \mathrm{g}$ ) ever reported and the $\mathrm{Na}_{2 / 3}\left[\mathrm{Mn}_{0.72} \mathrm{Mg}_{0.28}\right] \mathrm{O}_{2}$

\footnotetext{
* matthieu.saubanere@umontpellier.fr marie-liesse.doublet@umontpellier.fr
}

electrode [21, 22] shows reversible anionic redox without $\mathrm{Na}$-excess, cationic disorder or $\mathrm{O}_{2}$ release. [22] Over the past 5 years experimental and theoretical studies have contributed to figure out the origin of anionic redox as arising from oxygen lone-pair states in the material electronic structure. [16, 20, 23-25] However, debates and controversial interpretations persist in the literature on the reversibility of the anionic process upon charge/discharge. Yet, these questions are paramount to decide whether the objective of high-energy-density batteries can be achieved with Arich-TMOs materials.

This paper proposes a unified picture of anionic redox in Arich-TMOs, irrespectively of their structural-type and electronic structure. Based on rigorous concepts of electronic (band) structure theory, we investigate the impact of the material electronic ground state, A-excess, $\mathrm{M} / \mathrm{M}$ ' chemical substitution and cationic order/disorder on the electrochemical activity of the O-network. This conceptual approach is validated by first-principles calculations through the computation of the Electron Localization Function (ELF). [57] ELF is a topological tool of the electron density that probes the regions of space where paired-electrons are confined, and is used, for the first time in Arich-TMOs, to demonstrate the participation of oxygen lone-pairs in the redox activity of these electrodes. Within this framework, we identify the charge-transfer gap of TMOs $\left(\Delta_{C T}\right)$ and the number of O-holes per oxygen $\left(h^{O}\right)$ as pertinent descriptors to quantify the anionic capacity achievable in charge and its reversibility in discharge. This provides experimentalists with measurable quantities to infer the overall electrochemical performance of their materials, and new rational recipes to exit the maze of anionic redox. 


\section{Oxygen Lone-Pairs in A-Rich TMOs}

The participation of oxygens in the electrochemical activity of Arich-TMOs is now widely accepted as arising from $O(2 p)$ lone-pairs, [16, 20, 23-25] hereafter denoted $O_{2 p}$. How many oxygen lone-pairs occur in these materials can be straightforwardly deduced from the universal octet rule, which states that oxygen needs four electron pairs in its valence shell to be stable (see Supplementary Information S1). In layered or rocksalt Arich-TMOs, of general formulation $\mathrm{A}\left[\mathrm{A}_{x} \mathrm{M}_{1-x}\right] \mathrm{O}_{2}$, the average number of $\mid O_{2 p}$ per oxygen, $n$, is directly linked to the $x$ stoichiometry, which also sets the cationic order/disorder in the structure. Accordingly, $\mathrm{LiMO}_{2}, \mathrm{Li}_{2} \mathrm{MO}_{3}$ and $\mathrm{Li}_{5} \mathrm{MO}_{6}$ are cation-ordered phases, as $n$ is an integer for $x=0,1 / 3$ and $2 / 3$ (see Fig. 1 ). For $x \neq 0,1 / 3,2 / 3, n$ is fractional and cationic disorder [23,27] or crystal site discrimination $[25,28]$ is required to split the O-network into different O-sublattices, each of them recovering an integer but different $n$. Finally, $x>2 / 3$ implies unconventional $n>2$ number and TM oxidation states which may lead to unfavorable situations where the TM cations become more electronegative than the anions, therefore preventing the formation of the oxide phase. This analysis already surmises that any anionic oxidation inevitably violates the octet rule, so that cationic migrations and/or O-O pairing will be neccessary to compensate for the oxygen destabilization.

Cationic $\mathrm{M} / \mathrm{M}$ ' substitutions are levers to increase the number of $\mid O_{2 p}$ lone-pairs. As a robust criteria, we consider that all metals forming peroxide phases (e.g. alkali, alkali-earth or divalent $d^{10} \mathrm{TMs}$ ) have strong enough reducing powers to prevent M'-O bonding, therefore increasing $n$. Such substitutions might be seen as $\mathrm{A} / \mathrm{A}^{\prime}$ rather than $\mathrm{M} / \mathrm{M}^{\prime}$ as they lower the material total capacity when $\mathrm{A} \neq \mathrm{A}^{\prime}$. Cationic vacancies also increase $n$. This explains why anionic redox can be activated in materials showing no A-excess, as exemplified by the $\mathrm{Na}_{2 / 3}\left[\mathrm{Mn}_{0.72} \mathrm{Mg}_{0.28}\right] \mathrm{O}_{2}[21,22]$ and $\mathrm{Na}_{4 / 7}\left[\square_{1 / 7} \mathrm{Mn}_{6 / 7}\right] \mathrm{O}_{2}$ electrodes [28] (see Fig. 2). For M' being a $\mathrm{d}^{m<10}$ transition metal or a post-transitional metal, $n$ is set by the $\left(\mathrm{M}+\mathrm{M}^{\prime}\right)$ stoichiometry and is invariant with the substitution ratio. In Li-rich NMC $[19,29-33]$ or $\operatorname{Li}_{2}\left[\operatorname{Sn}_{y} \mathrm{M}_{1-y}\right] \mathrm{O}_{3} \quad(\mathrm{M}=\mathrm{Ru}, \mathrm{Ir})$ electrodes, $[4,8]$ the anionic capacity increases with $y$ due to the electrochemically inactive M' and not to the increase of $n$.

\section{Anionic Activity in A-Rich TMOs}

From now on, we will term "anionic activity" an oxidation process involving the $\mid O_{2 p}$ lone-pair states. Whether these states participate in the electrochemical activity of Arich-TMOs depends on the material electronic ground-state. According to the ZaanenSawatzky-Allen classification, [34] TMOs are described in a Mott-Hubbard $(U<\Delta)$ or charge-transfer regime

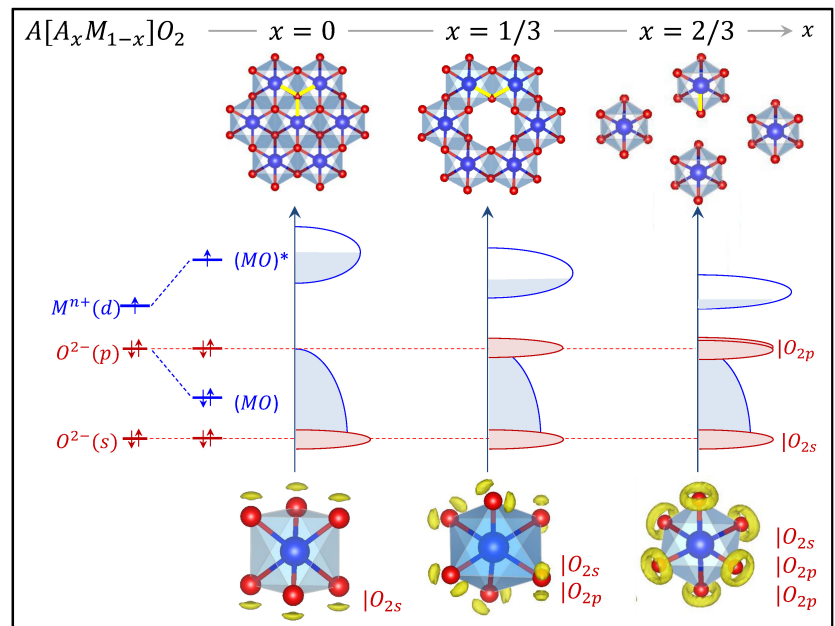

FIG. 1. Oxygen Lone-Pair Count: Schematic electronic structures for $\mathrm{LiMO}_{2}(x=0), \mathrm{Li}_{2} \mathrm{MO}_{3}(x=1 / 3)$ and $\mathrm{Li}_{5} \mathrm{MO}_{6}$ $(x=2 / 3)$ where the $\mid O_{2 s}$ and $\mid O_{2 p}$ lone-pair states are highlighted by red bands. In these structures, the average number of TM around each oxygen is 3,2 and 1, respectively, thus leaving $n=0,1$ and $2 \mid O_{2 p}$ lone-pairs per oxygen in each structure. For all $x$, the oxygen $2 s$-orbital makes negligible symmetry-allowed interactions with the metal $d$-orbitals and forms low-lying nearly non-bonding states. This leads to one $\mid O_{2 s}$ lone-pair per oxygen which is too deep in energy to participate in the electrochemical reaction. Electron Localization function (ELF) computed within the DFT+U framework for $\mathrm{LiCoO}_{2}, \mathrm{Li}_{2} \mathrm{RuO}_{3}$ and $\mathrm{Li}_{5} \mathrm{OsO}_{6}$ phases confirm the number of $\mid \mathrm{O}_{2 s}$ and $\mid \mathrm{O}_{2 p}$ lone-pairs per oxygen expected from the octet rule (see Section Methods).

$(U>\Delta)$ depending on the amplitude of the M-O charge transfer $(\Delta)$ with respect to the coulombic interactions in the $d$-shell $(U)$. The electronic regime of Arich-TMOs thus defines the amount of capacity available for the anionic process and is the first important criterion to consider when investigating the electrochemical properties of these electrodes. In the following, the anionic activity will be discussed separately for the two regimes, in the specific case of single-TM oxides. The concepts and rules established in this framework will then be extended to the general case of disordered and substituted TMOs, in order to build a unified picture of anionic redox.

\section{Mott-Hubbard Systems}

Mott-Hubbard systems are described by a partiallyfilled metallic band, splitted by the $U$ coulombic term and lying above the fully-filled anionic band. As shown in Fig. 3, the extraction of $\mathrm{e}^{-}$and $\mathrm{Li}^{+}$from the structure leads to an electrostatic stabilization of the (M-O)* states and a concomitant electrostatic destabilization of the $\mid O_{2 p}$ states for the oxygens lying in the vicinity of the $\mathrm{A}^{+}$vacancy. As long as the metallic states remain above the anionic states, the oxidation corresponds to a cationic process which is generally fully reversible. 


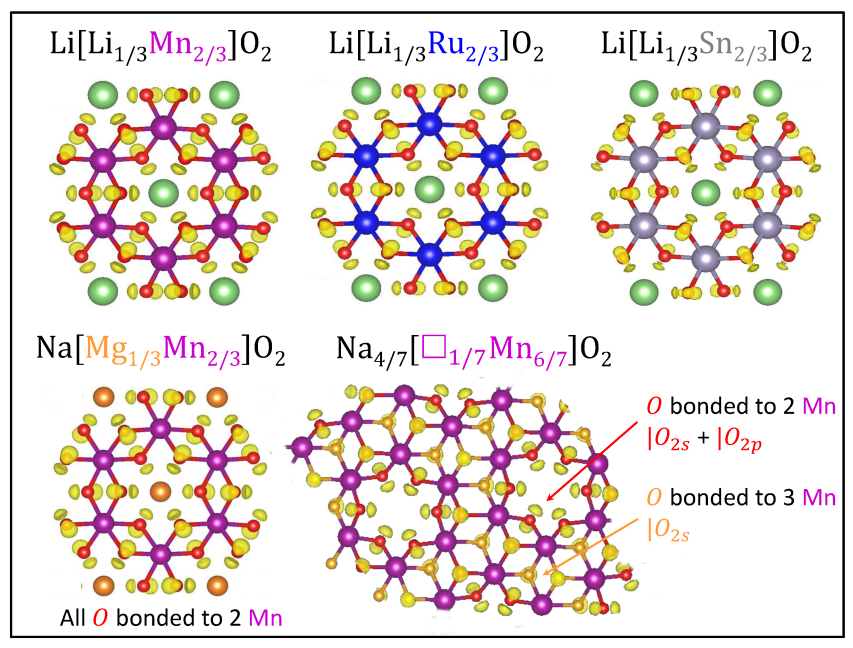

FIG. 2. Electron Localization Function computed from first-principles $\mathrm{DFT}+\mathrm{U}$ calculations (isovalues of 0.7 ) for $\mathrm{Li}\left[\mathrm{Li}_{1 / 3} \mathrm{M}_{2 / 3}\right] \mathrm{O}_{2} \quad(\mathrm{M}=\mathrm{Mn}, \mathrm{Ru}, \mathrm{Sn})$ showing an equivalent number of oxygen lone-pairs per oxygen (one $\mid O_{2 s}$ and one $\left.\mid O_{2 p}\right)$, irrespectively of the M-O bond covalency. For $\mathrm{Na}_{2 / 3}\left[\mathrm{Mg}_{1 / 3} \mathrm{Mn}_{2 / 3}\right] \mathrm{O}_{2}$, the apparent $\mathrm{Mn} / \mathrm{Mg}$ substitution in fact corresponds to a $\mathrm{Na} / \mathrm{Mg}$ substitution $\left(\mathrm{Mg}^{2+}\right.$ acting as $2 \mathrm{Na}^{+}$in the charge compensation) so that the material stoichiometry is $x=1 / 3$ (" $\mathrm{A}_{2} \mathrm{MO}_{3}$ "). For $\mathrm{Na}_{4 / 7}\left[\square_{1 / 7} \mathrm{Mn}_{6 / 7}\right] \mathrm{O}_{2}$, one and two oxygen lone-pairs per oxygen occur in the Mnrich (orange) and Mn-poor (red) O-environments, respectively.

When the two states get close in energy, the electronic ground-state of the material is (quasi)degenerate which is unstable towards any structural distortion that removes the degeneracy. The distortion must lower the crystal symmetry to allow efficient interactions between (M-O)* and $\mid O_{2 p}$, both states having different symmetries in the undistorted structure. It corresponds to the formation of a three-centered $\mathrm{M}-(\mathrm{O}-\mathrm{O})$ species, through a so-called Reductive Coupling Mechanism (RCM) [4, 24] which opens a gap in the material electronic structure. Interestingly, the transition metal initially oxidized is being reduced when the structural reorganization takes place, due to a redistribution of the $\mid O_{2 p}$ electrons into the new $\mathrm{M}-(\mathrm{O}-\mathrm{O})$ bonding states (highlighted in orange in Fig. 3). The participation of the anions in the electrochemical activity of Arich-TMOs is then activated through the dynamic of the cationic process ( $\mathrm{O}$ to $\mathrm{M}$ charge transfer). As a proof of concept, we computed the ELF isovalue for a typical Mott-Hubbard system, namely the $\beta-\mathrm{Li}_{2-z} \mathrm{IrO}_{3}$ model electrode. [9] As shown in Fig. 3, one $\mid O_{2 p}$ per oxygen is lost in the delithiated structure, concomitantly with the appearence of weak O-O bonds (see Supplementary Information S2 for details).

\section{Charge Transfer Systems}

Charge-transfer systems are described by an empty

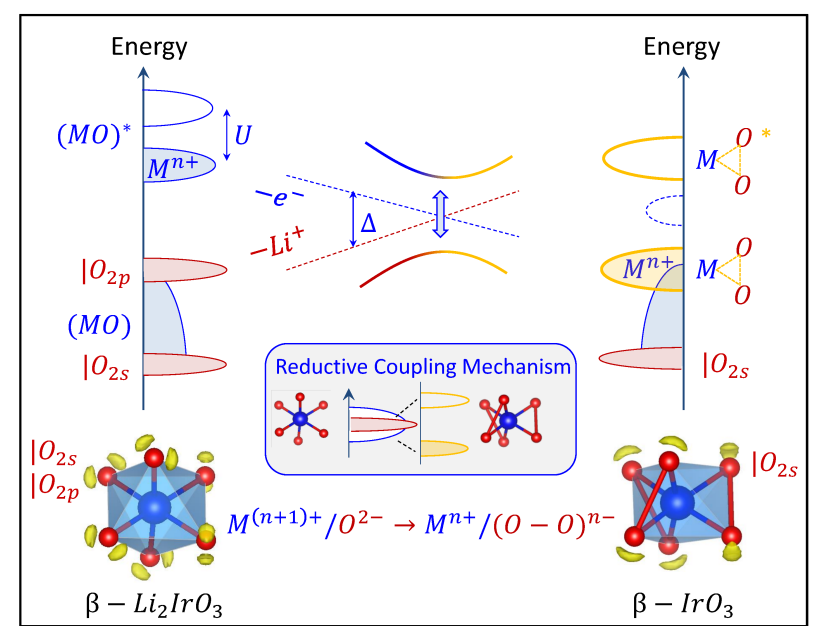

FIG. 3. Dynamics of A-removal in Mott-Hubbard Systems. Qualitative electronic structure of Arich-TMOs displaying a Mott-Hubbard electronic ground state (left). The electrostatic stabilization (resp. destabilization) of the (M$\mathrm{O})^{*}$ antibonding states (resp. $\mid O_{2 p}$ states) upon $e^{-}$(resp. $\mathrm{Li}^{+}$) removal is shown by the dashed lines. The reductive coupling mechanism (grey inset) lifts the electronic degeneracy into $\mathrm{M}-(\mathrm{O}-\mathrm{O})$ bonding and antibonding bands represented in orange, which induces a $\mid O_{2 p}$ to $(\mathrm{M}-\mathrm{O})^{*}$ charge transfer. In discharge, the re-insertion of $\mathrm{Li}$ should involve the $(\mathrm{M}-\mathrm{O}-$ $\mathrm{O}))^{*}$ states hence allowing the system the return back to the undistorted structure through a perfectly reversible mechanism. Note that if all $\mathrm{M}(d)$ states are not involved in the $\mathrm{RCM}$, a fraction of $\mathrm{M}(d)$-states (dotted blue band) may remain in the RCM-gap, therefore leading to some irreversibility in discharge. The participation of the $O_{2 p}$ states in the oxidation process is illustrated for the $\beta-\mathrm{Li}_{2-z} \mathrm{IrO}_{3}$ electrode which was recently reported as exhibiting a mixed cationic-anionic redox activity. The ELF value computed for the lithiated $(z=0)$ and delithiated $(z=2)$ phases clearly shows the loss of one oxygen lone-pair per oxygen upon oxidation.

metallic band lying above the fully-filled anionic band (here the $\mid O_{2 p}$ band) and separated in energy by the charge-transfer gap $\Delta_{C T}$ (see Fig. 4). The removal of electrons from the $\mid O_{2 p}$ localized states generates unstable oxygens that must recombine to recover a stable electronic configuration (octet rule). As a result of O-O pairing, the narrow $\mid O_{2 p}$ band splits into $\sigma, \pi, \pi^{*}$ and $\sigma^{*}$ discrete bands with an amplitude as large as the $\mathrm{O}-\mathrm{O}$ distance is short, i.e. as the $(\mathrm{O}-\mathrm{O})^{n-}$ species are oxidized (red and yellow triangles in Fig. 4). As a robust criteria, a given $(\mathrm{O}-\mathrm{O})^{n-}$ dimer is stable as long as its highest occupied states lie below the lowest unoccupied metallic band. Accordingly, the amplitude of $\Delta_{C T}$ with respect to $\mathrm{O}^{2-} /\left(\mathrm{O}_{2}\right)^{2-}, \mathrm{O}^{2-} /\left(\mathrm{O}_{2}\right)^{-}$or $\mathrm{O}^{2-} / \mathrm{O}_{2}$ redox energies is the pertinent descriptor to predict the amount of extra-capacity achievable in charge and its reversibity in discharge.

Following the horizontal blue line of Fig. $4,(\mathrm{O}-\mathrm{O})^{n-}$ species are stable as long as $\Delta_{C T}>\Delta_{O-O}^{\sigma}$ (before (1)). As for the reductive coupling mechanism discussed above, 
the anionic oxidation is fully reversible in discharge, since the electronic states involved in oxidation and reduction are similar. From (1) to (2) the metallic band lies between the $\pi^{*}$ and $\sigma^{*}$ bands, so that $\left(\mathrm{O}_{2}\right)^{2-}$ peroxides can form. As a result of the $\sigma^{*} / \mathrm{M}(d)$ band inversion, a cationic reduction is expected in the first-step discharge, leading to voltage hysteresis. The peroxide reduction in a second-step discharge obviously depends on the capacity used for the cationic process. Above (2) the metallic band lies below the $\pi^{*}$-states of the oxidized species $\left(\Delta_{O-O}^{\pi}>\Delta_{C T}\right)$. This results in the release of $\mathrm{O}_{2}$ gas concomitantly with a cationic reduction, through a mechanism known as a reductive elimination. [35]

Remarkably, the weak occurrence of transition metal peroxides reported in the literature [36, 37] suggests that most TMOs display $\Delta_{C T}$ band gaps lower than the $\mathrm{O}^{2-} /\left(\mathrm{O}_{2}\right)^{2-}$ redox energy (below (1)). In contrast, alkali or alkali-earth oxides display much larger $\Delta_{C T}$ and are known to form peroxides. This strongly suggests that $\left(\mathrm{O}_{2}\right)^{2-}$ peroxides in charged Arich-TMOs should only occur in $\mathrm{M}(d)$-free $/ \mathrm{A}(s, p)$-rich O-environments, as exemplified in the $\mathrm{Ba}_{5} \mathrm{Ru}_{2} \mathrm{O}_{11}$ peroxide/oxide phase. [38] In this structure, the $\sigma^{*}$ states of peroxides $\left(d_{O-O}=1.5\right.$ $\AA$ ) are raised in energy far above the metallic band, thus preventing their reduction in discharge.

\section{The Unified Picture of Anionic Redox}

All mechanisms described above can be put together to build a unified picture of anionic redox in Arich-TMOs. In this general framework, the number of holes per oxygen, $h^{O}$, is shown to be the critical parameter governing the reversibility of the anionic process.

In the most general case of Arich-TMOs combining cationic disorder and chemical substitutions, the oxygen network consists in a statistical distribution of various $\mathrm{O}$-sublattices, each of them having a different number of $\mid O_{2 p}$ lone-pairs due to different A/M/M' local environments. $O_{2 p}$ states being non-bonding, their energy is primarily dictated by the electrostatic field exerted by the surrounding cationic charges. The lower the cationic charge around one oxygen, the smaller its Madelung potential and the higher in energy its $\mid O_{2 p}$ states in the electronic structure. How many holes are generated upon charge on each O-sublattice thus depends (i) on the relative energy of the electrochemically active O-sublattices, i.e. how homogeneous is the O-network, and (ii) on the material electronic ground-state, i.e. how much of the total theoretical capacity is available for the anionic process. Knowing this, the overall electrochemical behavior of Arich-TMOs can be deduced from the superimposition of each O-sublattice individual activity, accounting for their statistical weight in the pristine material, and following the mechanisms described above. This approach is illustrated in Fig. 5 for a representative set of $\mathrm{A}\left[\mathrm{A}_{x} \mathrm{M}_{1-x}\right] \mathrm{O}_{2}$ showing different $\mathrm{A} / \mathrm{M} / \mathrm{M}$ ' compositions and distributions and a theoretical capacity corresponding to $(1+x)$ electrons. The important message of this

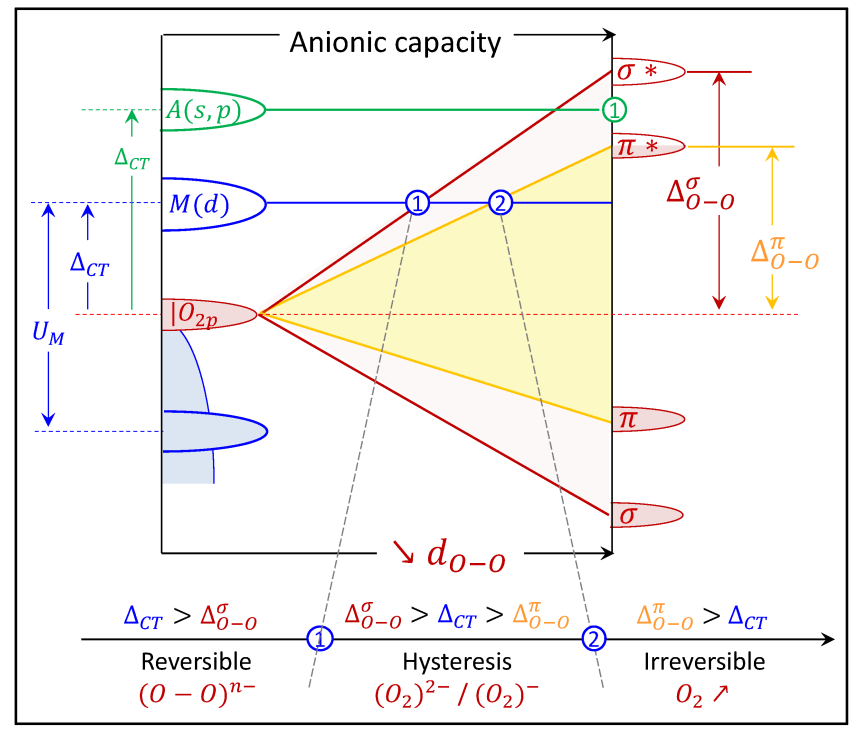

FIG. 4. Dynamics of the oxidation process in chargetransfer Arich-TMOs. Qualitative electronic structure of Arich-TMOs displaying a charge transfer electronic groundstate in the reduced phase (left). The splitting of the $\mid O_{2 p}$ narrow band into discrete $\sigma, \pi, \pi^{*}$ and $\sigma^{*}$ states is highlighted by the red $\left(\Delta_{O-O}^{\sigma}\right)$ and yellow $\left(\Delta_{O-O}^{\pi}\right)$ triangles, as a function of the decreasing $\mathrm{O}-\mathrm{O}$ distance (bottom horizontal axis) and the increasing oxidation state of the $(\mathrm{O}-\mathrm{O})^{n-}$ species (top horizontal axis). The different domains of anionic activity are represented by the intervals delimited by (1) and (2). The (1) to (2) narrow domain highlights the weak ability of transition metals to stabilize true peroxides in their structure $\left(\Delta_{C T}\right.$ generally smaller than the $\mathrm{O}^{2-} /\left(\mathrm{O}_{2}\right)^{2-}$ redox energy), in contrast to $\mathrm{A}(s, p)$ metals showing much larger $\Delta_{C T}$ gaps in the oxide phases.

figure is that $h^{O} \leq x$ is required for the anionic process to be reversible. Above this critical value, the anionic oxidation in charge is partially irreversible in discharge, except for O-sublattices having $\mathrm{M}(d)$-free/A-rich local environments for which a reversible $\mathrm{O}^{2-} /\left(\mathrm{O}_{2}\right)^{2-}$ redox pair can exist.

For homogeneous O-networks, $h^{O} \leq x$ implies that at least $1-x$ electron of the total capacity is consumed by a preceding cationic process involving one or more electro-active metals (Fig. 5a). The voltage profile of these materials should display nicely reversible electrochemical processes (see Fig.6a) and a potential drop between the cationic and anionic reactions, as the signature of two distinct $\mathrm{M}^{n+} / \mathrm{M}^{(n+1)+}$ and $\mathrm{O}^{2-} /(\mathrm{O}-\mathrm{O})^{2(2-x)-}$ redox pairs. The anionic process should be associated with a larger polarization than the cationic process, due to the structural reorganization of the O-network. [39] This electrochemical behavior is exemplified by the tridimensional $\beta-\mathrm{Li}_{2} \mathrm{IrO}_{3}$ electrode [9] showing well-defined reversible plateaus upon charge/discharge, no voltage hysteresis and limited irreversible capacity. 3D structures should be favored over 2D structures in which cationic migration is triggered by the interlayer electrostatic in- 


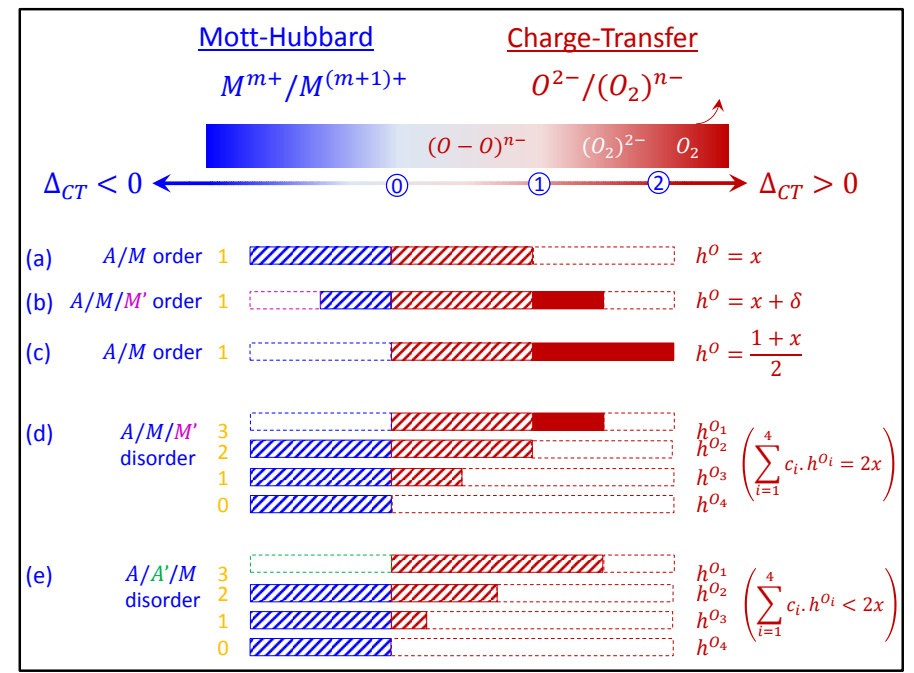

FIG. 5. Unified picture of anionic redox in ArichTMOs. Cationic and anionic processes expected for $\mathrm{A}\left[\mathrm{A}_{x} \mathrm{M}_{1-x}\right] \mathrm{O}_{2}(0<x \leq 1 / 3)$ showing different $\mathrm{A} / \mathrm{A}^{\prime} / \mathrm{M} / \mathrm{M}^{\prime}$ compositions and distributions. The cationic and anionic processes are represented by blue and red rectangles, respectively, the area of which being correlated to reversible (dashed) and irreversible (plain) capacities. Mott-Hubbard and chargetransfer regimes are distinguished by a negative and positive $\Delta_{C T}$, respectively. M' refers to an electrochemically inactive metal having no impact on the number of $\mid O_{2 p}$ lone-pairs in the structure (pink M' = strongly correlated TM with halffilled $d$-band, post-transitional metal or $\mathrm{d}^{0}$ metal). A' refers to an electrochemically inactive metal/vacancy increasing the number of $\mid O_{2 p}$ lone-pairs in the structure (green $\mathrm{A}^{\prime}=\mathrm{A}(s, p)$ mono- or divalent metal or metal vacancy). The number of oxygen lone-pairs per oxygen is indicated in yellow and $h^{O}$ is calculated for each system by substracting the cationic capacity to the total capacity. For disordered materials, the statistical weight of each O-sublattices is represented by the $c_{i}$ coefficients. The total theoretical capacity is equivalent for all examples $(1+x$ electrons) except for the last example in which the $\mathrm{A}\left[\mathrm{A}_{x} \mathrm{M}_{1-x}\right] \mathrm{O}_{2}$ general formulation is no longer valid due to the $\mathrm{A} / \mathrm{A}^{\prime}$ substitution.

stability at low A-content. This phenomenon was shown to limit the reversible capacity of the layered $\alpha-\mathrm{Li}_{2} \mathrm{IrO}_{3}$ electrode [8] compared to its 3D $\beta$-polymorph. [9] Favoring electronic delocalization in 2D structures through the increase of bond covalency or with temperature should contribute against this instability. This is supported by the series of TM-chalcogenides $\left(\mathrm{MX}_{2}\right.$ or $\mathrm{MX}_{3}, \mathrm{X}=\mathrm{S}$, Se, $\mathrm{Te})[40,41]$ showing stable layered structures and by the layered $\mathrm{Li}_{2} \mathrm{RuO}_{3}$ electrode showing full reversible capacity when cycled at $50^{\circ} \mathrm{C}$. [42]

Any M/M' chemical substitution for an electrochemically inactive M' metal invariably leads to partial irreversibility of the anionic process due to the increase of $h^{O}$ above the critical $x$ value (Fig. 5b). Above $x$, the highly oxidized O-network should disproportionate into $h^{O}$-related fractions of $\mathrm{O}^{2-},\left(\mathrm{O}_{2}\right)^{2-}$ and $\mathrm{O}_{2}$ sublattices with relative weights set by the chemical composition

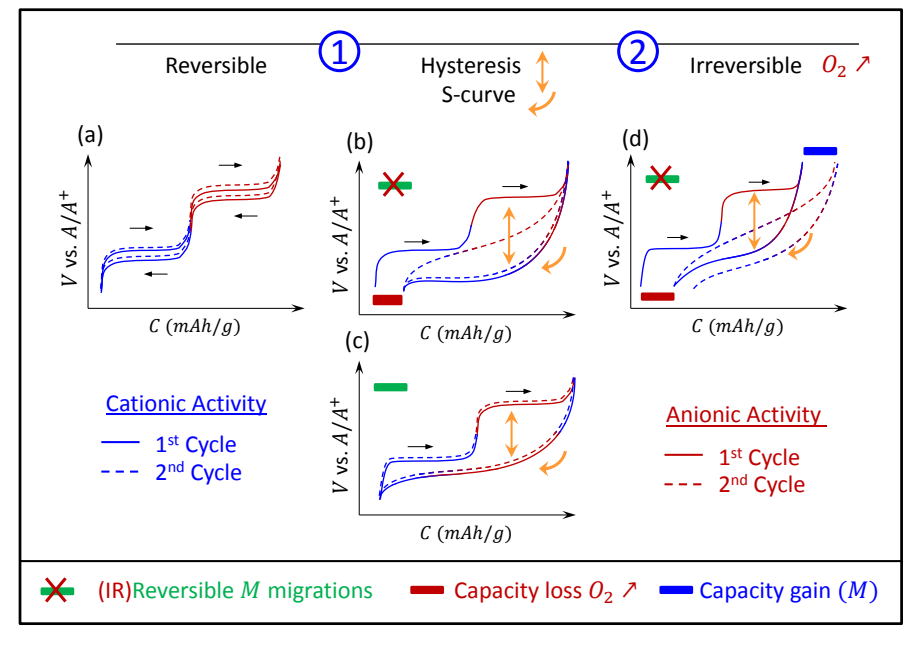

FIG. 6. Schematic galvanostatic curves of A-Rich TMOs. Illustration of the cationic (blue) and anionic (red) processes in A-rich TMOs for the different scenarios given in Figs. 3 and 4. (a) Fully reversible cationic and anionic process with no $\mathrm{O}_{2}$ release and no cation migration in charge. (b)-(c)-(d) The first part of the discharge corresponds to a cationic activity (blue) due to $\mathrm{O}_{2}$ release at high-charging. This leads to a hysteresis between charge/discharge (yellow). When $\mathrm{O}_{2}$ gas release occurs in first charge due to cationic migration/disorder, the process is not reversible leading to a persistent hysteresis in further cycles and a S-shape curve due to the multiplication of TM and (O-O) redox centers (b). The plateaus in charge are recovered if no $\mathrm{O}_{2}$ release occurs at high charging and if cationic migrations are fully reversible in discharge (c). The capacity loss in charge due to $\mathrm{O}_{2}$ gas release (red rectangle) is partially compensated in discharge (blue rectangle) by the activation of a novel TM redox couple (d).

of the pristine structure. Cationic migrations should take place from the critical $h^{O}=x$ state-of-charge to assist the disproportionation. Oxygens having more cationic vacancies in their local environment will be primarily oxidized, and prone to $\mathrm{O}_{2}$ gas release through the partial reduction of the surrounding transition metal. An hysteresis is expected in the voltage profile of the electrode and the total capacity achieved in charge cannot be fully recovered in discharge (see Fig.6b). Most Arich-TMOs reported in the literature belong to this category. They correspond to the general formulation $\mathrm{A}\left[\mathrm{A}_{x}\left(\mathrm{M}_{1-y} \mathrm{M}_{y}^{\prime}\right)_{1-x}\right] \mathrm{O}_{2}$ with $\mathrm{M}^{\prime}=d^{0}$, half-filled $d$-band TM with large $U$ splitting (e.g. $\mathrm{Mn}^{4+}, \mathrm{Fe}^{3+}$ ), or post-transitional (e.g. $\mathrm{Sn}^{4+}, \mathrm{Te}^{6+}$ ) metal. These electrodes display large voltage hysteresis, cationic migration and a capacity loss linearly correlated to $y$, as exemplified by the series of $\operatorname{Li}_{2}\left[\mathrm{Ru}_{1-y} \mathrm{M}_{y}^{\prime}\right] \mathrm{O}_{2}\left(\mathrm{M}^{\prime}=\mathrm{Sn}\right.$, $\mathrm{Ti}, \mathrm{Mn})[4,5,43]$ and $\mathrm{Li}_{2}\left[\operatorname{Ir}_{1-y} \mathrm{Sn}_{y}\right] \mathrm{O}_{2}$. [8] Note that, in rare cases, the TM reduction induced by $\mathrm{O}_{2}$ release may activate a cationic redox-pair in discharge that was not accessible in first charge. This could allow recovering, at least partially, the first-charge capacity in further cycles (see Fig. 6c). Reaching the complete substitution 
of $\mathrm{M}$ for an inactive $\mathrm{M}$ ' metal leads to charge-transfer materials, such as $\mathrm{Li}_{2} \mathrm{MnO}_{3}$, $[10,44]$ in which the theoretical capacity is fully supported by the anions, i.e. $h^{O}=(1+x) / 2$. Unless $x$ is decreased to activate a cationic redox-pair, $[45,46]$ charge-transfer TMOs should display poor electrochemical performance [10] associated with a progressive decomposition of the electrode upon cycling, persistent voltage hysteresis and capacity fade in the voltage profile (see Fig. 6d). Finally, chemical A/A' substitutions with A' being highly reducing metals (large $\Delta_{C T}$ ) appears as the only way to achieve a reversible anionic capacity with $h^{O}>x$ (see Fig. 5e) due to the possible stabilization of peroxides $\left(h^{O}=1\right)$ in $\mathrm{M}(d)$-free/A'-rich environments. However, the statistical weight of this O-sublattice must be significant to benefit to the anionic capacity, which implies large A/A' substitution ratio $y$, i.e. low capacity and poor electronic conductivity.

Noteworthy, any release of $\mathrm{O}_{2}$ gas in charge lowers $h^{O}$ in further cycles. Consequently, Arich-TMOs can display nicely reversible electrochemical cycles after a first charge that kills the activity of the most unstable O-sublattices. This is exemplified for instance by the $\mathrm{Li}_{1.2}\left[\mathrm{Mn}_{0.54} \mathrm{Co}_{0.13} \mathrm{Ni}_{0.13}\right] \mathrm{O}_{2}$ [3] and $\mathrm{A}_{1.3} \mathrm{Nb}_{0.3} \mathrm{Mn}_{0.4} \mathrm{O}_{2}$ $(\mathrm{A}=\mathrm{Li}, \mathrm{Na})$ electrodes $[47,48]$ sustaining reversible capacity of $\sim 250$ and $\sim 300 \mathrm{mAh} / \mathrm{g}$, respectively. Interestingly, the lithiated $\mathrm{Li}_{1.3} \mathrm{Nb}_{0.3} \mathrm{Fe}_{0.4} \mathrm{O}_{2}$ electrode shows significantly lower performance compared to its Mn-based homologue. While this behavior was attributed by the authors to a Fe-O bond covalency larger than Mn-O, [48] our unified picture shows it arises from different $h^{O}$. Alike $\mathrm{Li}_{2} \mathrm{MnO}_{3}, \mathrm{Li}_{1.3} \mathrm{Nb}_{0.3} \mathrm{Fe}_{0.4} \mathrm{O}_{2}$ is a charge-transfer insulator (inactive $\mathrm{Fe}^{3+}$ ions confirmed by DFT calculations in ref. [48]) for which the total theoretical capacity is fully supported by the anions $\left(h^{O}=(1+x) / 2\right)$. In the Mn-based system, 0.4 electrons are consumed by a preceding $\mathrm{Mn}^{3+} / \mathrm{Mn}^{4+}$ cationic process, leading to significantly lower $h^{O}$ and better cycling performance, after the first cycle. The same comparison can be made between the $\mathrm{Li}_{2} \mathrm{MnO}_{3}[10]$ and the Mn-defective $\mathrm{Na}_{4 / 7}\left[\square_{1 / 7} \mathrm{Mn}_{6 / 7}\right] \mathrm{O}_{2}$ electrodes. [28] While the former displays no cationic redox and highly irreversible anionic redox, the latter shows nicely reversible cationic and anionic redox processes, very similar to the $\beta-\mathrm{Li}_{2} \mathrm{IrO}_{3}$ electrode [9] thanks to a limited extra-capacity $\left(h^{O}<x\right)$. Finally, approaching the limits of cationic and anionic electrochemical activity with the Li-rich layered rocksalt $\mathrm{Li}_{3} \mathrm{IrO}_{4}(x=1 / 2)$ electrode shows that the delithiated $\mathrm{IrO}_{4}$ electrode converts into $\mathrm{IrO}_{3}$ at the end of charge, which limits $h^{O}$ to $1 / 3$ in further cycles. [25] Overall, these examples highlight the negligible role of M-O bond covalency in the electrochemical behavior of Arich-TMOs and suggest that $h^{O}=1 / 3$ is the upper limit for achieving reversible anionic capacity in $\mathrm{A}\left[\mathrm{A}_{x} \mathrm{M}_{1-x}\right] \mathrm{O}_{2}$ electrodes. Obviously, any chemical substitution lowering $h^{O}$ through the activation of a cationic redox-pair, is a good strategy to favor structural stability and more reversible anionic electro-activity, as nicely exemplified by transition metal oxyfluorides. [27, 49]

\section{CONCLUSION}

So far, most Arich-TMOs reported in the literature as displaying high-energy-density due to anionic redox, are prone to structural instabilities that prevent their use in practical applications. Several alternatives have been proposed to limit or suppress these irreversibilities, among which the increase of M-O bond covalency, the chemical substitution of $\mathrm{M}$ for $d^{0}$ metals or the use of cation-disordered rocksalt structures. The unified picture developed in this paper contrasts with these statements in showing that the number of holes per oxygen $\left(h^{O}\right)$ is the critical parameter to sustain a reversible anionic capacity. From a representative set of materials reported in the literature, $h^{O}=1 / 3$ appears as the critical value to avoid $\mathrm{O}_{2}$ release and achieve fully reversible anionic redox. Measurable quantities such as the TMO band gap $\left(\Delta_{C T}\right)$ and the $x$ stoichiometry are therefore sufficient to determine $h^{O}$ and to infer the electrochemical behavior of $\mathrm{A}\left[\mathrm{A}_{x} \mathrm{M}_{1-x}\right] \mathrm{O}_{2}$ electrodes.

Within this general framework, the best candidates for high-energy-density are those cumulating an optimal cationic capacity and an anionic capacity limited to $h^{O} \leq 1 / 3$. Tri-dimensional structures should be preferred over layered structures to prevent structural instability at low A-content and take advantage of the full theoretical capacity. Ordered structures should favor homogeneous O-networks should avoid h Very few TMOs should satisfy these requirements, in particular when they also have to meet industrial specifications such as cost, toxicity and natural abundance. Overall, strategies devoted to the activation of anionic redox as a lever to improve the energy density of electrode materials are risky as they implicitly means that anions become less electronegative than cations at some point, therefore questioning the structural stability of the materials. The triptych of high-potential, high-capacity and high structural stability still appears out of our current reach and calls for trade-offs.
[1] Padhi, A. K., Nanjundaswamy, K. S., Masquelier, C., Okada, S. \& Goodenough, J. B. Effect of structure on the $\mathrm{Fe}^{3+}-\mathrm{Fe}^{2+}$ redox couple in iron phosphates. J. Electrochem. Soc. 144, 1609-1613 (1997). 
[2] Saubanère, M., Ben Yahia, M., Lebègue, S. \& Doublet, M.-L., Nat. Commun. 55559 (2014).

[3] Koga, H., Croguennec, L., Ménétrier, M., Douhil, K., Belin, S., Bourgeois, L., Suard, E., Weil, F. \&Delmas, C., Reversible oxygen participation to the redox processes revealed for $\mathrm{Li}_{1.20} \mathrm{Mn}_{0.54} \mathrm{Co}_{0.13} \mathrm{Ni}_{0.13} \mathrm{O}_{2}$. J. Electrochem. Soc. 160 A786-A792 (2013).

[4] Sathiya, M. et al. Reversible anionic redox chemistry in high-capacity layered-oxide electrodes. Nat. Mater. 12 827-835 (2013).

[5] Sathiya, M., Ramesha, K., Rousse, G., Foix, D., Gonbeau, D., Prakash, A. S., Doublet, M.-L. \& Tarascon, J.-M., High performance $\mathrm{Li}_{2} \mathrm{Ru}_{1-y} \mathrm{Mn}_{y} \mathrm{O}_{3}$ cathode materials for rechargeable lithium-ion batteries: their understanding. Chem. Mater. 25 1121-1131 (2013).

[6] Luo, K. et al. Charge-compensation in 3d-transitionmetal-oxide intercalation cathodes through the generation of localized electron holes on oxygen. Nat. Chem. 8 684-691 (2016).

[7] Yabuuchi, N. et al. High-capacity electrode materials for rechargeable lithium batteries: Li3NbO4-based system with cation-disordered rocksalt structure. Proc. Nat. Acad. Sci. 112(25), 7650-7655 (2015).

[8] McCalla, E. et al. Visualization of $\mathrm{O}-\mathrm{O}$ peroxo-like dimers in high-capacity layered oxides for Li-ion batteries. Science 350, 1516-1521 (2015).

[9] Pearce, P.E. et al. Evidence for anionic redox activity in a tridimensional-ordered Li-rich positive electrode $\beta$ $\mathrm{Li}_{2} \mathrm{IrO}_{3}$. Nat. Mater. 16 580-587 (2017).

[10] Robertson, A. D. \& Bruce, P. G., Mechanism of Electrochemical Activity in $\mathrm{Li}_{2} \mathrm{MnO}_{3}$. Chem. Mater. 15, 19841987 (2003)

[11] Ito, D., Liu, Y., Chong, S. \& Wu, Y.-F., Cyclic deterioration and its improvement for Li-rich layered cathode material $\mathrm{Li}\left[\mathrm{Ni}_{0.17} \mathrm{Li}_{0.2} \mathrm{Co}_{0.07} \mathrm{Mn}_{0.56} \mathrm{O}_{2}\right.$. J. Power Sources 195, 567-573 (2010).

[12] Croy, J. R., Iddir, H., Gallagher, K., Johnson, C. S., Benedeck, R., Balasubramanian, M., First-charge instabilities of layered-layered lithium-ion-battery materials. Phys. Chem. Chem. Phys. 17, 24382 (2015).

[13] Gent, W. E. et al. Coupling between oxygen redox and cation migration explains unusual electrochemistry in lithium-rich layered oxides. Nat. Commun. 8, 2091-1-12 (2017).

[14] Lee, E. \& Persson, K. A., Structural and Chemical Evolution of the Layered Li-Excess $\mathrm{Li}_{x} \mathrm{MnO}_{3}$ as a Function of Li Content from First-Principles Calculations. Adv. Energy Mater. 4, 1400498 (2014).

[15] Qian, D., Xu, B., Chic, M. \& Meng, Y. S., Uncovering the roles of oxygen vacancies in cation migration in lithium excess layered oxides. Phys. Chem. Chem. Phys. 16 14665-14668 (2014).

[16] Xie, Y., Saubanère, M., Doublet, M.-L., Requirements for reversible extra-capacity in Li-rich layered oxides for Li-ion batteries. Energy Env. Sci. 10, 266-274 (2017).

[17] Urban, A., Matts, I., Abdellahi, A. \& Ceder, G., Computation Design and Preparation of Cation-Disordered Oxides for High-Energy-Density Li-Ion Batteries. Adv. Ener. Mater. 1600488-1-8 (2016).

[18] Li, B. \& Xia, D., Anionic Redox in Rechargeable Lithium Batteries. Adv. Mater. 29 1701054-1-28 (2017).

[19] Li, W., Song, B. \& Manthiram, A., High-voltage positive electrode materials for lithium-ion batteries. Chem. Soc. Rev. 46, 3006-3059 (2017).
[20] Assat, G. \& Tarascon, J.-M., Fundamental understanding and practical challenges of anionic redox activity in Li-ion batteries. Nature Energy 3 376-286 (2018).

[21] Yabuuchi, N., Hara, R., Kubota, K., Paulsen, J., Kumakurad \& S., Komaba, S., A new electrode material for rechargeable sodium batteries: P2-type $\mathrm{Na}_{2 / 3}\left[\mathrm{Mg}_{0.28} \mathrm{Mn}_{0.72} \mathrm{O}_{2}\right.$ with anomalously high reversible capacity. J. Mater. Chem. A 2, 16851-16855 (2014).

[22] Maitra, U. et al. Oxygen redox chemistry without excess alkali-metal ions in $\mathrm{Na}_{2 / 3}\left[\mathrm{Mg}_{0.28} \mathrm{Mn}_{0.72}\right]_{O} 2$. Nature Chemistry 10 288-295 (2018).

[23] Seo, D.-H.,J., Urban, A., Malik, R., Kang, S. Y. \& Ceder, G., The structural and chemical origin of the oxygen redox activity in layered and cation-disordered Li-excess cathode materials. Nature Chemistry 8, 692-697 (2016).

[24] Saubanère, M., McCalla, E., Tarascon, J.-M. \& Doublet, M.-L., The intriguing question of anionic redox in highenergy density cathodes for Li-ion batteries. Energy Env. Sci. 9, 984-991 (2016).

[25] Perez., A. J., Jacquet, Q., Batuk, D., Iadecola, A., Saubanère, M., Rousse, G., Larcher, D., Vezin, H., Doublet, M.-L. \& Tarascon, J.-M., Approaching the limits of cationic and anionic electrochemical activity with the Li-rich layered rocksalt $\mathrm{Li}_{3} \mathrm{IrO}_{4}$. Nat. Energy 2, 954-962 (2017).

[26] Becke, A. D. \& Edgecombe, K. E., A simple measure of electron localization in atomic and molecular systems. $J$. Chem. Phys. 92 5397-5403 (1990).

[27] House, R., Jin, L., Maitra, U., Tsuruta, K., Somerville, J., Forstermann, D., Massel, F., Duda, L., Roberts, M. R. \& Bruce, P. G., Lithium manganese oxyfluoride as a new cathode material exhibiting oxygen redox. Energy $\&$ Environ. Sci. 11 926-932 (2018).

[28] Mortemard de Boisse, B., Nishimura, S.-I, Watanabe, E., Lander, L., Tsuchimoto, A., Kikkawa, J., Kobayashi, E., Asakura, D., Okubo, M. \& Yamada, A., Highly Reversible Oxygen-Redox Chemistry at $4.1 \mathrm{~V}$ in $\mathrm{Na}_{4 / 7-x}\left[X_{1 / 7} \mathrm{Mn}_{6 / 7}\right] \mathrm{O}_{2}$ (X: Mn Vacancy). Adv. Energy Mater. 20181800409 1-7.

[29] Hong, J., Xiao, D.-D., Zhang, X.-D., Yin, Y.-X., Guo, Y.-G., Gu, L. \& Wan L.-J., Review - Lithium-excess layered cathodes for lithium rechargeable batteries. $J$. Electrochem. Soc. 162, A2447-A2467 (2015).

[30] Rozier, P. \& Tarascon, J. M., Review — Li-rich layered oxide cathodes for next-generation Li-ion batteries: chances and challenges. J. Electrochem. Soc. 162, A2490A2499 (2015).

[31] Croy, J. R., Balasubramanian, M., Gallagher, K. G. \& Burrell, A. K. Review of the U. S. Department of Energy's 'Deep Dive' effort to understand voltage fade in Li- and Mn-rich cathodes. Acc. Chem. Res. 48, 28132821 (2015).

[32] Zheng, J., Myeong, S., Cho, W., Yan, P., Xiao, J., Wang, C., Cho, J. \& Zhang, J.-G., Li- and Mn-rich cathode materials: challenges to commercialization. Adv. Energy Mater. 7, 1601284 (2016).

[33] Hy, S., L. H., Zhang, M., Qian, D., Hwang, B.-J. \& Meng, Y. S., Performance and design considerations for lithium excess layered oxide positive electrode materials for lithium ion batteries. Energy Environ. Sci. 9, 19311954 (2016).

[34] Zaanen, J., Sawatzky, G. A. \& Allen, J. W. Band gaps and electronic structure of transition-metal compounds. Phys. Rev. Lett. 55, 418-421 (1985). 
[35] Crabtree, R. H., The Organometallic Chemistry of the Transition Metals. Third Edition. (John-Wiley \& Sons, Inc., New-York, 2001).

[36] Toriumi, K. \& Saito, Y. Electron-Density Distribution in Crystals of $\alpha-\mathrm{K}_{2} \mathrm{CrO}_{4}$. Acta Cryst. B 34 3149-3156 (1978).

[37] Fischer, J., Veillard, A. \& Weiss, R. Theoretica Chimica Acta 24 317-333 (1972).

[38] Grimaud, A. et al. Chemical Activity of the Peroxide/Oxide Redox Couple: Case Study of $\mathrm{Ba}_{5} \mathrm{Ru}_{2} \mathrm{O}_{11}$ in Aqueous and Organic Solvents. Chem. Mater. 30 38823893 (2018).

[39] Assat, G., Foix, D., Delacourt, C., Iadecola, A., Dedryvère, R. \& Tarascon, J.-M., Fundamental interplay between anionic/cationic redox governing the kinetics and thermodynamics of lithium-rich cathodes. Nat. Commun. 82219 (2017).

[40] Rouxel, J., Anion-cation redox competition and the formation of new compounds in highly covalent systems. Chem. Eur. J. 2 1053-1059 (1996).

[41] Rouxel, J., Some solid state chemistry with holes: anioncation redox competition in solids. Curr. Sci. 73 31-39 (1997).

[42] Yabuuchi, N., Material Design Concept of LithiumExcess Materials with Rocksalt-related Structures for Rechargeable Non-Aqueous Batteries. The Chem. Record 18, 1-19 (2018).

[43] Sathiya, M. et al. Origin of voltage decay in high-capacity layered oxide electrodes. Nat. Mater. 14 230-238 (2015).

[44] Kalyani, P., Chitra, S., Mohan, T. \& Gopukumar, S., Lithium metal rechargeable cells using $\mathrm{Li}_{2} \mathrm{MnO}_{3}$ as the positive electrode. J. Power Sources, 80, 103-106 (1999).

[45] Armstrong, A. R., Robertson, A. D., Bruce, P. G., Overcharging manganese oxides: Extracting lithium beyond $\mathrm{Mn}^{4+}$. J. Power Sources 146, 275-280 (2005).

[46] Freire, M., Lebedev, O.I., Maignan, A., Jordi, C \& Pralong V., Nanostructured $\mathrm{Li}_{2} \mathrm{MnO}_{3}$ : a disordered rock salt type structure for high energy density Li ion batteries. $J$. Mater. Chem. A 5 21898-21902 (2017).

[47] Sato., K., Nakayama, M., Glushenkov, A. M., Mukai, T., Hashimoto, Y., Yananka, K., Yishimura, M., Ohta, T. \& Yabuuchi, N., Na-Excess Cation-Disordered Rocksalt Oxide: $\mathrm{Na}_{1.3} \mathrm{Nb}_{0.3} \mathrm{Mn}_{0.4} \mathrm{O}_{2}$. Chem. Mater. 29 5043-5047 (2017).

[48] Yabuuchi, N. et al. Origin of stabilization and destabilization in solid-state redox reaction of oxide ions for lithiumion batteries. Nat. Commun. 7:13814 (2016).

[49] Lee, J. et al. Reversible $\mathrm{Mn}^{2+} / \mathrm{Mn}^{4+}$ double redox in lithium-excess cathode materials. Nature 556 185-190 (2018).

\section{Methods}

Spin-polarized calculations were performed using the VASP code $[50,51]$ within the density functional theory (DFT) framework. Perdew-Burke-Ernzerhof (PBE) functional [52] with a generalized gradient approximation form (GGA-PBE) was adopted to treat the exchange correlation energy. The rotationally invariant Dudarev method (DFT+U) [53] was applied to account for strongly correlated $d$ electrons with different $\mathrm{U}_{\text {eff }}$ values. The range-separated hybrid functional [54] (HSE06) was also used for a sake of comparison with $\mathrm{DFT}+\mathrm{U}$. To obtain a good numerical sampling of the electron densities in Brillouin zone, the Monkhorst-Pack technique [55] was used. Moreover, with the application of the projector augmented wave (PAW) technique, [56] the plane-wave energy cutoff was determined to be $600 \mathrm{eV}$. For structural relaxations, all atomic coordinates and lattice parameters were fully relaxed until the force acting on each atom is less than $3 \cdot 10^{-3} \mathrm{eV} . \AA^{-1}$.

ELF: A pertinent tool to characterize and visualize $\mid O$ lone-pairs in a material is known as the Electron Localization Function (ELF). To the best of our knowledge, this tool has never been used in A-rich TMOs, although $O$ lone-pairs are central in the rationalization of their electrochemical properties. It is a topological tool of the electron density that pinpoints the regions of space where paired electrons are confined. Initially designed by Becke and Edgecombe for atoms and molecules, [57] it was developed in the Density Functional Theory framework by Savin [58] and is becoming popular in material science due to its holistic approach to electronic structures. [59] ELF measures the Pauli repulsion through the probability of finding an electron of spin $\sigma$ in the vicinity of another electron of same spin. The explicit formulation is:

$$
E L F=\left[1+\left\{\frac{K(\mathbf{r})}{K_{h}[\rho(\mathbf{r})]}\right\}^{2}\right]^{-1}
$$

where $K$ is the curvature of the electron-pair density $\gamma_{2}\left(r_{1}^{\sigma}, r_{2}^{\sigma}\right), \rho(\mathbf{r})$ the electron density and $K_{h}$ the value of $K$ for the homogeneous electron gas with dentity $\rho$.

The ELF values were computed for the different materials given as examples in the paper, at various $\mathrm{Li} / \mathrm{Na}-$ contents. ELF values range from 0 (no paired electrons) to 1 (paired electrons) and are here plotted for 0.7 isovalues in Fig. 1, Fig. 2 and Supplementary Information Fig.S1, Fig.S2.

\section{Data Availability}

The datasets generated during and/or analysed during the current study are available from the corresponding author on reasonable request.

\section{References}

[50] Kresse, G. \& Furthmüller, J., Efficiency of ab-initio total energy calculations for metals and semiconductors using a plane-wave basis set. Computational Materials Science 6 15-50 (1996).

[51] Kresse, G. \& Hafner, J., Ab initio molecular dynamics for liquid metals. Phys. Rev. B 47 558-561 (1993). 
[52] Perdew, J. P., Burke K. \& Ernzerhof, M., Generalized Gradient Approximation Made Simple. Phys. Rev. Lett. 77 3865-3868 (1996).

[53] Dudarev, S. L., Botton, G. A., Savrasov, S. Y., Humphreys, C. J. \& Sutton, A. P., Electron-energy-loss spectra and the structural stability of nickel oxide: An LSDA+U study. Phys. Rev. B 57 1505-1509 (1998).

[54] Heyd, J., Scuseria, G. E. \& Ernzerhof, M., Hybrid functionals based on a screened Coulomb potential. $J$. Chem. Phys. 118 8207-8210 (2003).

[55] Monkhorst, H. J. \& Pack, J. D., Special points for Brillouin-zone integrations. Phys. Rev. B 13 51885192 (1976).

[56] Blöchl, P. E., Projector augmented-wave method. Phys. Rev. B 50 17953-17979 (1994).

[57] Becke, A. D. \& Edgecombe, K. E. A simple measure of electron localization in atomic and molecular systems. J. Chem. Phys. 92 5397-5403 (1990).

[58] Savin, A., Jepsen, O., Flad, J., Andersen, O. K., Preuss, H. \& von Schnering, H. G., Electron Localization in Solid-state Structures of the Elements: the Diamond Structure. Angew. Chem. Int. Ed. Engl. 31 187-188 (1992).
[59] Causà, M.; D'Amore, M.; Gentile, F.; Menendez, F.; Calatayud, M. Electron Localization Function and Maximum Probability Domains analysis of semi-ionic oxides crystals, surfaces and surface defects. Computational and Theoretical Chemistry 1053, 315-321 (2015).

\section{Acknowledgements}

The authors acknowledge the $\mathrm{RS}_{2} \mathrm{E}$ institution and the Agence Nationale pour la Recheche (ANR) - DeliRedox $\mathrm{n}^{\circ}$ ANR-14-CE05-0020 - for supporting this work.

\section{Author Contributions}

All authors contributed equally to the DFT calculations \& analyses. MS and MLD developed the theoretical framework and wrote the paper.

\section{Additional Information}

Supplementary information is available in the online version of the paper. Reprints and permissions information is available online at www.nature.com/reprints. Correspondence and requests for materials should be addressed to M.-L.D or M.S.

\section{Competing financial interests}

The authors declare no competing financial interests. 